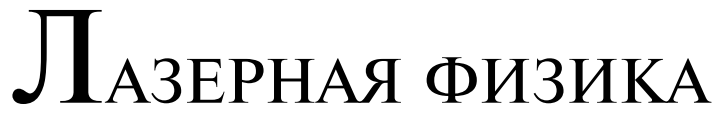

\section{LASER PHYSICS}

УДК 621.373 .533 .9

\section{ПРОСТРАНСТВЕННОЕ РАСПРЕАЕЛЕНИЕ ПЛОТНОСТИ ИОННОГО ПОТОКА В ААЗЕРНО-ПЛАЗМЕННОМ ИСТОЧНИКЕ ААЯ НАНЕСЕНИЯ НАНОПОКРЫТИЙ НА ПОАЛОЖКИ УВЕАИЧЕННЫХ РАЗМЕРОВ}

\author{
В. К. ГОНЧАРОВ ${ }^{1)}$, М. В. ПУЗЫРЕВ ${ }^{1)}$, \\ В. Ю. СТУПАКЕВИЧ ${ }^{2)}$ Н. И. ШУЛЬГАН \\ ${ }^{1)}$ Институт прикладных физических проблем им. А. Н. Севченко БГУ, \\ ул. Курчатова, 7, 220045, г. Минск, Беларусь \\ ${ }^{2)}$ Гродненский государственный университет им. Янки Купаль, \\ ул. Ожешко, 22, 230023, г. Гродно, Беларусь \\ ${ }^{3)}$ Белорусский государственный университет, пр. Независимости, 4, 220030, г. Минск, Беларусь
}

Выполнено экспериментальное определение равномерности плотности ионных потоков на подложку увеличенных размеров $\left(\sim 200 \mathrm{~cm}^{2}\right)$ в целях формирования наноструктур лазерно-плазменным методом. Отмечено, что система для осаждения наноструктур состоит из эрозионного лазерного факела материала мишени и подложки, расположенных в вакуумной камере. Для плавной регулировки параметров наносимых на подложку частиц между лазерной мишенью и подложкой установлена сетка, на которую подается отрицательный по отношению к лазерной мишени потенциал. В результате после сетки формируется поток частиц, состоящий преимущественно из ионов, энергией которых можно надежно и плавно управлять, подавая на сетку положительный по отношению

Образец цитирования:

Гончаров ВК, Пузырев МВ, Ступакевич ВЮ, Шульган НИ. Пространственное распределение плотности ионного потока в лазерно-плазменном источнике для нанесения нанопокрытий на подложки увеличенных размеров. Журнал Белорусского государственного университета. Физика. 2021; 2:81-87.

https://doi.org/10.33581/2520-2243-2021-2-81-87

\section{For citation:}

Goncharov VK, Puzyrev MV, Stupakevich VYu, Shulhan NI. The spatial density distribution of the ion flux in the laser-plasma source for deposition of nanocoating on substrates of increased size. Journal of the Belarusian State University. Physics. 2021;2:81-87. Russian.

https://doi.org/10.33581/2520-2243-2021-2-81-87

\begin{abstract}
А в торы:
Виктор Константинович Гончаров - доктор физико-математических наук, профессор; главный научный сотрудник лаборатории лазерной плазмодинамики.

Михаил Валентинович Пузырев - кандидат физико-математических наук, доцент; заведующий лабораторией лазерной плазмодинамики.

Валерий Юзефович Ступакевич - старший преподаватель кафедры информационных систем и технологий физикотехнического факультета.

Никита Игоревич Шульган - студент факультета радиофизики и компьютерных технологий. Научный руководитель - В. К. Гончаров.
\end{abstract}

\section{Authors:}

Victor K. Goncharov, doctor of science (physics and mathematics), full professor; chief researcher at the laboratory of laser plasma dynamics.

Michail V. Puzyrev, $\mathrm{PhD}$ (physics and mathematics), docent; head of the laboratory of laser plasma dynamics.

puzyrev@bsu.by

Valery Yu. Stupakevich, senior lecturer at the department of information systems and technologies, physico-technical faculty. tvsad@grsu.by

Nikita I. Shulhan, student at the faculty of radiophysics and computer technologies.

nikita.shulgan@gmail.com 
к подложке потенциал. Проведенные эксперименты показали, что однородность плотности ионных потоков на подложку увеличенных размеров ( $\left.200 \mathrm{~cm}^{2}\right)$ в лазерно-плазменном источнике для нанесения нанопокрытий можно повысить, подавая на подложку ускоряющий потенциал (по отношению к сетке). Минимальная разница между плотностью ионного потока в центре и на краю мишени составляет 5 \%. В результате технологически возможно производить очистку поверхности подложки ионами материала лазерной мишени (вторичная эмиссия), создавать псевдодиффузионный слой материала мишени в приповерхностной области подложки и наносить на подложку материал лазерной мишени. При этом все перечисленные операции можно выполнять последовательно, не разгерметизируя вакуумную камеру, что позволит получить нанопокрытия с высокой адгезией и на подложках увеличенных размеров.

Ключевые слова: лазерная плазма; ионные пучки; наноструктуры; высокая адгезия.

\title{
THE SPATIAL DENSITY DISTRIBUTION OF THE ION FLUX IN THE LASER-PLASMA SOURCE FOR DEPOSITION OF NANOCOATING ON SUBSTRATES OF INCREASED SIZE
}

\author{
V. K. GONCHAROV ${ }^{\mathrm{a}}$, M. V. PUZYREV ${ }^{\mathrm{a}}$, V. YU. STUPAKEVICH ${ }^{\mathrm{b}}$, N. I. SHULHAN \\ ${ }^{\mathrm{a}}$ A. N. Sevchenko Institute of Applied Physical Problems, Belarusian State University, \\ 7 Kurčatava Street, Minsk 220045, Belarus \\ ${ }^{\mathrm{b}}$ Yanka Kupala State University of Grodno, 22 Ažeška Street, Hrodna 230023, Belarus \\ ${ }^{\mathrm{c}}$ Belarusian State University, 4 Niezalieznasci Avenue, Minsk 220030, Belarus \\ Corresponding author: M.V.Puzyrev (puzyrev@bsu.by)
}

The present work is devoted to the experimental determination of the uniformity of the ion flux density on a substrate with an increased size $\left(\sim 200 \mathrm{~cm}^{2}\right)$ in order to form nanostructures by the laser-plasma method. The system for deposition of nanostructures consists of an erosion laser torch of the target material and a substrate located in a vacuum chamber. For smooth adjustment of the parameters of the deposited particles on the substrate, a grid is located between the laser target and the substrate, on which a negative potential is applied relative to the laser target. As a result, a particle stream is formed after the grid, consisting mainly of ions, whose energy can be reliably and smoothly controlled by applying a positive potential to the grid in relation to the substrate. Experiments have shown that the uniformity of the density of ion fluxes on a substrate of increased size $\left(-200 \mathrm{~cm}^{2}\right)$ in a laser-plasma source for nanocoating can be increased by applying an accelerating potential to the substrate in relation to the grid. The minimum difference between the ion flux density in the center of the target and at its edge can be reduced to $\sim 5 \%$. As a result, it is technologically possible to clean the surface of the substrate with ions of the laser target material (secondary emission), create a pseudodiffusion layer of the target material in the near-surface region of the substrate, and apply the laser target material to the substrate. At the same time, all these operations can be performed sequentially without depressurising the vacuum chamber. This allows obtaining coating with good adhesion on substrates of increased size.

Keywords: laser plasma; ion beams; nanostructures; high adhesion.

\section{Введение}

Одним из наиболее перспективных методов формирования наноструктур является лазерно-плазменный метод. Он обладает рядом преимуществ. Прежде всего это стерильность, возможность получать плазму из веществ, находящихся в любом агрегатном состоянии (в том числе тугоплавких материалов), экологическая чистота процесса.

Нанесение нанопленок на различные материалы обычно производится в вакууме. В этом случае внутри вакуумной камеры лазерная мишень и подложка располагаются параллельно друг другу, а лазерное излучение направляется через окно вакуумной камеры под некоторым углом к поверхности мишени. Эрозионный лазерный факел истекает в полупространство, и часть продуктов разрушения лазерной мишени попадают на поверхность подложки. В результате данного процесса на поверхности подложки образуются нанопленки материала лазерной мишени [1-3]. Для получения нанопленок с различными параметрами необходимо изменять режимы их нанесения. Это достигается путем смены режимов частотно-импульсного лазера. И если управление частотой лазера и числом импульсов легко обеспечить в автоматическом режиме, то осуществлять регулировку плотности мощности лазерного излучения на поверхности лазерной 
мишени достаточно сложно. Динамический диапазон изменения энергии отдельного лазерного импульса в современных частотных технологических лазерах невелик, а для повышения плотности мощности воздействующего на лазерную мишень излучения за счет фокусировки требуется применение достаточно сложных оптико-механических систем, которые, как правило, не обладают большой надежностью.

Для более точного изменения энергии частиц напыляемого вещества можно использовать подачу электрического потенциала в промежутке мишень - подложка. Однако при разлете эрозионного лазерного факела в вакууме в плазме факела формируется двойной электрический слой. Впереди летят наиболее энергичные электроны, а за ними - ионы [4]. Подача дополнительного электрического потенциала на промежуток мишень - подложка приводит к его взаимодействию с полем двойного электрического слоя. При этом в плазме эрозионного лазерного факела формируются сложные колебания и неустойчивый режим, что затрудняет управление энергией плазменных частиц.

В работе [5] было предложено между лазерной мишенью и подложкой поместить сетку, подав на нее отрицательный по отношению к мишени потенциал. В этом случае после сетки удается получить поток заряженных частиц, состоящий преимущественно из ионов. Подавая на подложку отрицательный по отношению к сетке потенциал, можно плавно регулировать энергию ионов и производить нанесение нанопокрытий материала мишени на поверхность подложки за счет потока ионов.

Были выполнены работы по изучению режимов нанесения нанопокрытий различных материалов лазерной мишени на поверхность подложек из разных материалов [6].

Однако эти эксперименты проводились с использованием подложек малых размеров $\left(\sim 10 \mathrm{~cm}^{2}\right)$. На практике часто возникает необходимость в нанесении нанопокрытий на достаточно большие поверхности. Для этого нужно найти режимы работы лазерно-плазменного источника, при которых можно получить равномерные нанопокрытия на подложках увеличенных размеров.

Настоящая работа посвящена исследованию пространственного распределения плотности ионного потока на подложку в форме круга площадью 200 см².

\section{Оборудование и методики экспериментов}

На алюминиевую мишень, расположенную в вакуумной камере при давлении около $2,6 \cdot 10^{-3}$ Па, воздействовали излучением лазера $\mathrm{Nd}^{3+}$ : YAG LS-2137 (Lotis TII, Беларусь - Япония). Плотность мощности лазерного излучения составила $3 \cdot 10^{8}$ Вт/см². При этом на промежутки мишень - сетка и сетка подложка подавались разные потенциалы $U_{1}$ и $U_{2}$. Измерения производились с помощью осциллографа TDS2022B (Tektronix, США). Общая схема эксперимента описана в работе [5]. Взаимное расположение мишени, сетки, подложки и лазерного луча значительно зависит от объема и формы вакуумной камеры. В настоящих экспериментах расстояние мишень - подложка составляло 12 см, а сетка располагалась посередине между ними, т. е. на расстоянии 6 см от поверхности мишени.

\section{Результаты и их обсуждение}

Так как в используемом лазерно-плазменном источнике эрозионный лазерный факел осесимметричен, то и ионный поток, сформированный после сетки и движущийся в сторону подложки, также осесимметричен. В связи с этим для контроля пространственного распределения плотности ионного потока на подложку достаточно контролировать изменение плотности ионного тока вдоль радиуса подложки.

Для этого перед подложкой были установлены два дополнительных зонда площадью $1,0 \times 0,5 \mathrm{~cm}^{2}$ каждый. Один из них являлся неподвижным и располагался в центре подложки, другой имел возможность передвижения вдоль радиуса подложки от центра к краю. Общая схема эксперимента представлена на рис. 1.

Так как использовался двухканальный осциллограф, то контроль электрических параметров элементов схемы (см. рис. 1) производился последовательно по два параметра.

Предварительно были проконтролированы токи в промежутках мишень - сетка и сетка - подложка в режиме формирования потоков заряженных частиц после сетки преимущественно в виде ионов. Результаты этих измерений представлены на рис. 2.

При этом на сетку подавался отрицательный по отношению к мишени потенциал 20 В, на подложку отрицательный по отношению к сетке потенциал 20 В. Результат данного эксперимента представлен на рис. 2. Как видно из рис. 2 , $a$, кривая тока имеет несколько «горбов». Это можно объяснить следующим образом. Появление плазмы в промежутке мишень - сетка при отрицательном по отношению к мишени потенциале сетки 20 В заставляет без организации двойного электрического слоя ионы двигаться к отрицательному потенциалу сетки, а электроны - к мишени. Однако из-за экранирующего действия ионов на потенциал сетки практически электроны движутся с небольшой скоростью к поверхности мишени. В это время ток в цепи сетка - мишень определяется в основном током ионов (первый «горб»).

$$
\text { 52Y - cmovemtera ncmopur yonexa }
$$




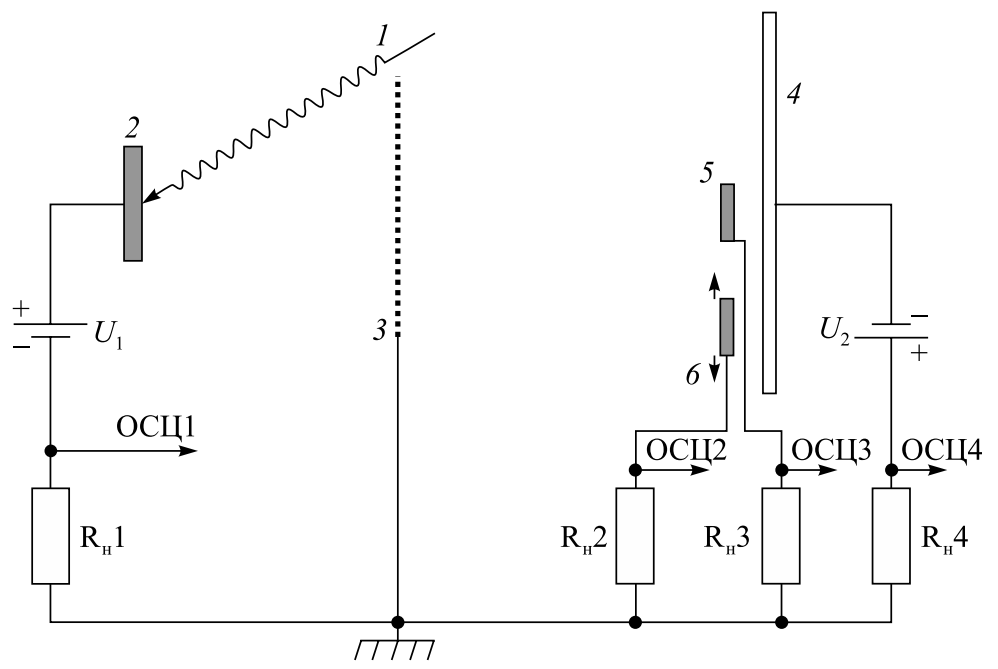

Puc. 1. Общая схема эксперимента:

1 - лазерное излучение; 2 - алюминиевая мишень; 3 - сетка;

4 - подложка; 5 - неподвижный зонд; 6 - подвижный зонд;

ОСЦ1 и ОСЦ2 - сигналы, снимаемые на первый луч; ОСЦ3 и ОСЦ4 - сигналы, снимаемые на второй луч; $U_{1}$ и $U_{2}$ - независимые источники питания;

$\mathrm{R}_{\mathrm{H}} 1, \mathrm{R}_{\mathrm{H}} 2, \mathrm{R}_{\mathrm{H}} 3$ и $\mathrm{R}_{\mathrm{H}} 4$ - нагрузочные сопротивления

Fig. 1. General scheme of the experiment:

1 - laser radiation; 2 - aluminum target; 3 - grid; 4 - substrate;

5 - fixed probe; 6 - mobile probe; ОСЦ1 and ОСЦ2 - signals taken on the first beam;

ОСЦ 3 and OCЦ4 - signals taken on the second beam; $U_{1}$ and $U_{2}$ - independent power sources; $\mathrm{R}_{\mathrm{H}} 1, \mathrm{R}_{\mathrm{H}} 2, \mathrm{R}_{\mathrm{H}} 3$ and $\mathrm{R}_{\mathrm{H}} 4$ - load resistance

$a / a$

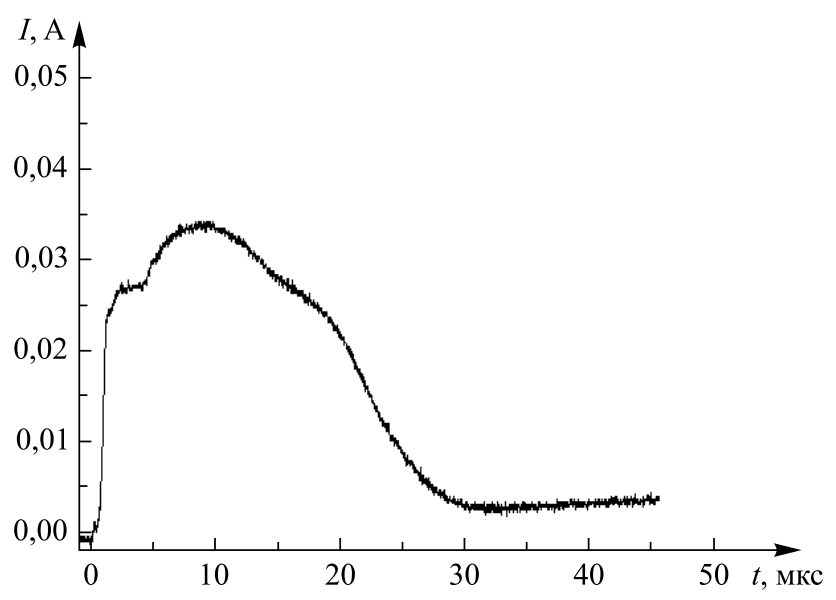

$\sigma / b$

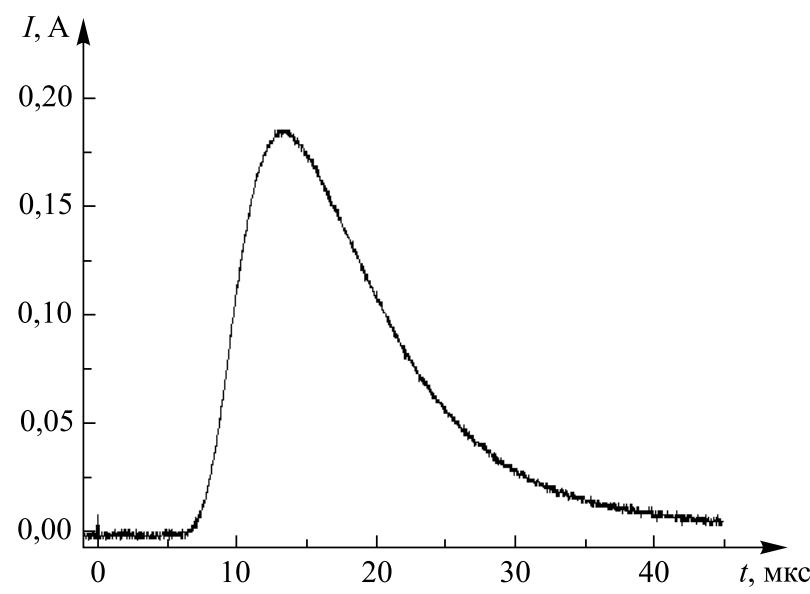

Puc. 2. Токи при воздействии лазерного излучения плотностью мощности $3 \cdot 10^{8} \mathrm{BT} / \mathrm{cm}^{2}$ на алюминиевую мишень: $a$ - в промежутке мишень - сетка; $\sigma$ - в промежутке сетка - подложка

Fig. 2. Currents at acting laser radiation with a power density of $3 \cdot 10^{8} \mathrm{~W} / \mathrm{cm}^{2}$ on aluminum target: $a$-in the target - grid interval; $b$ - in the grid - substrate interval

Как только основной поток ионов пролетел сетку ( 4 мкс после начала воздействия лазерного излучения), потенциал, поданный на нее с источника питания $U_{1}$, уже не заэкранирован ионами, и электроны начинают двигаться к мишени, резко увеличивая обратный ток. Результатом прямого тока ионов и обратного тока электронов на кривой тока в промежутке мишень - сетка является второй «горб».

Третий «горб» на кривой тока в промежутке мишень - сетка определяется ионным потоком и обратным потоком электронов в конце распадающегося лазерно-плазменного факела. При этом после сетки формируется поток заряженных частиц в сторону подложки, состоящий преимущественно из ионов (см. рис. 2, б). И это общий ток ионов на подложку увеличенных размеров. Необходимо экспериментально проконтролировать распределение плотности ионного потока вдоль радиуса подложки увеличенных размеров. 
Рассмотрим сигналы с неподвижного и подвижного зондов. Когда зонды находятся рядом в центpe подложки, сигналы практически совпадают. Это фактически калибровка каналов (рис. 3, a). Она осуществлялась расположением системы крепления относительно эрозионного лазерного факела двух зондов таким образом, чтобы сигналы с них были одинаковыми. Расстояние измерялось между центрами зондов. С учетом размеров зондов (1 см) ионный ток регистрировался на расстоянии от центра подложки вплоть до $8 \mathrm{~cm}$.

При удалении подвижного зонда от центра подложки сигнал на подвижном зонде задерживается во времени и может изменяться по амплитуде. Задержка объясняется тем, что при увеличении расстояния между неподвижным и подвижным зондами расстояние, которое проходит ионный поток на подвижный зонд, увеличивается (см. рис. 3,6$)$.

Так как в проведенных экспериментах использовался частотно-импульсный лазер с большим временем наработки, то уровень энергии в каждом импульсе может быть нестабильным. Для устранения влияния нестабильности лазера на результаты экспериментов выполнялись относительные измерения тока подвижного зонда $I_{2}$ к току неподвижного зонда $I_{1}$. Измерения $I_{2} / I_{1}$ были проведены по отношению площадей и по отношению максимальных амплитуд. Результаты оказались очень близки (рис. 4).

На рис. $4, a$, представлена кривая изменения отношений токов подвижного и неподвижного зондов вдоль радиуса подложки увеличенных размеров без подачи потенциала на промежуток сетка - подложка.

Из рисунка видно, что вдоль кривой наблюдается некоторая неравномерность. Она может обусловливаться неравномерностью лазерного излучения по пятну излучения и неравномерностью формы эрозионного пятна в зоне лазерного излучения. По этой причине ионный поток после сетки в отсутствие электрического потенциала в промежутке сетка - подложка определяется только кинетической энергией ионов за счет плазмодинамического давления в эрозионном лазерном факеле в промежутке мишень - сетка. Однако такой режим в лазерно-плазменном источнике нанесения нанопокрытий использовать нецелесообразно.

При подаче на сетку положительного по отношению к подложке потенциала 20 В (см. рис. 4, б) неоднородности уменьшаются за счет дополнительного более равномерного увеличения кинетической энергии в электрическом поле. Следует отметить, что в таких условиях обычно происходит напыление материала лазерной мишени на подложку [6].

При увеличении потенциала в промежутке сетка - подложка до 50 В наблюдается некоторое возрастание неоднородности плотности ионного потока вдоль радиуса подложки увеличенных размеров (см. рис. 4, в). Это можно объяснить неоднородностью вторичной ионной эмиссии с поверхности подложки, связанной с неоднородностью параметров поверхности подложки увеличенных размеров.

$a / a$

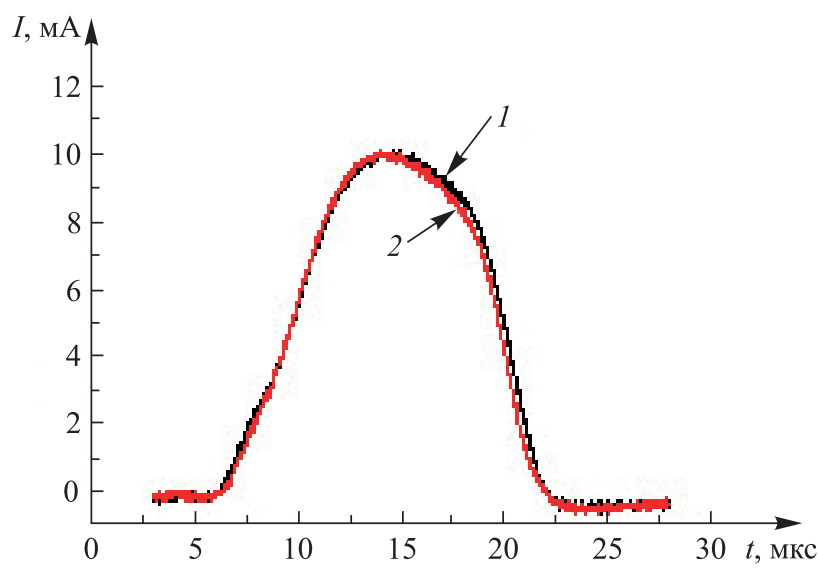

$\sigma / b$

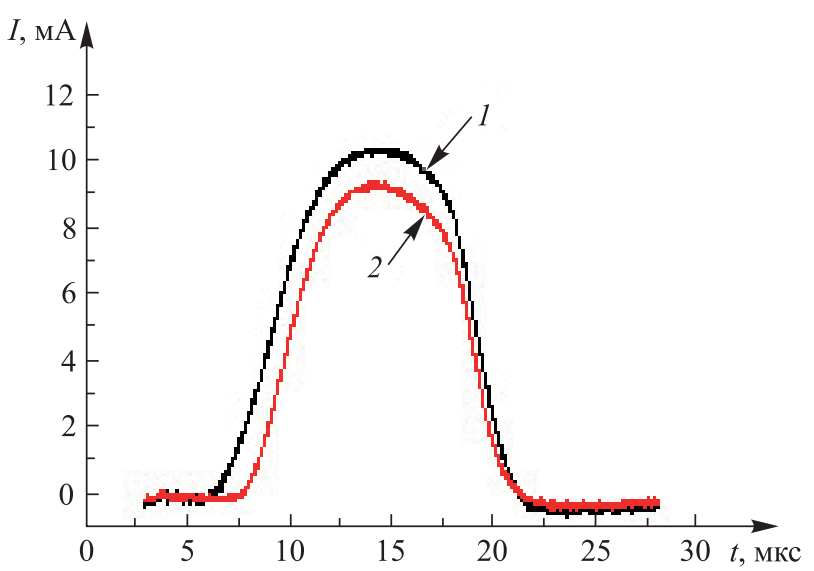

Puc. 3. Токи на зондах ( 1 - неподвижный зонд; 2 - подвижный зонд) при воздействии на алюминиевую мишень лазерного излучения плотностью мощности $3 \cdot 10^{8} \mathrm{BT} / \mathrm{cm}^{2}$ при потенциале

в промежутках мишень - сетка и сетка - подложка $20 \mathrm{~B}$ :

$a$ - расстояние между зондами $0 \mathrm{cm;} \sigma$ - расстояние между зондами $6 \mathrm{~cm}$

Fig. 3. Currents on the probes ( 1 - fixed probe; 2 - mobile probe)

at acting laser radiation on the aluminum target with a power density of $3 \cdot 10^{8} \mathrm{~W} / \mathrm{cm}^{2}$

at a potential in the target - grid interval and the grid - substrate interval of $20 \mathrm{~V}$ :

$a$-the distance between the probes is $0 \mathrm{~cm} ; b$ - the distance between the probes is $6 \mathrm{~cm}$

D2Y - cmovemtera ncmopur yonexa 

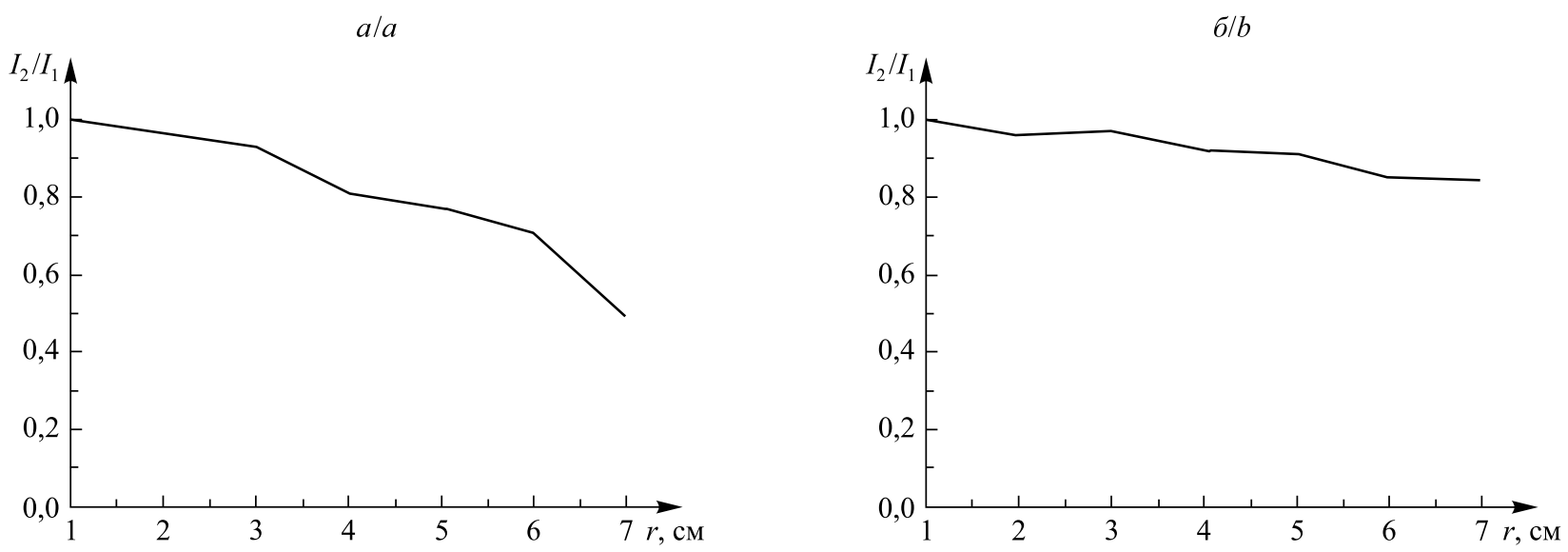

$B / c$
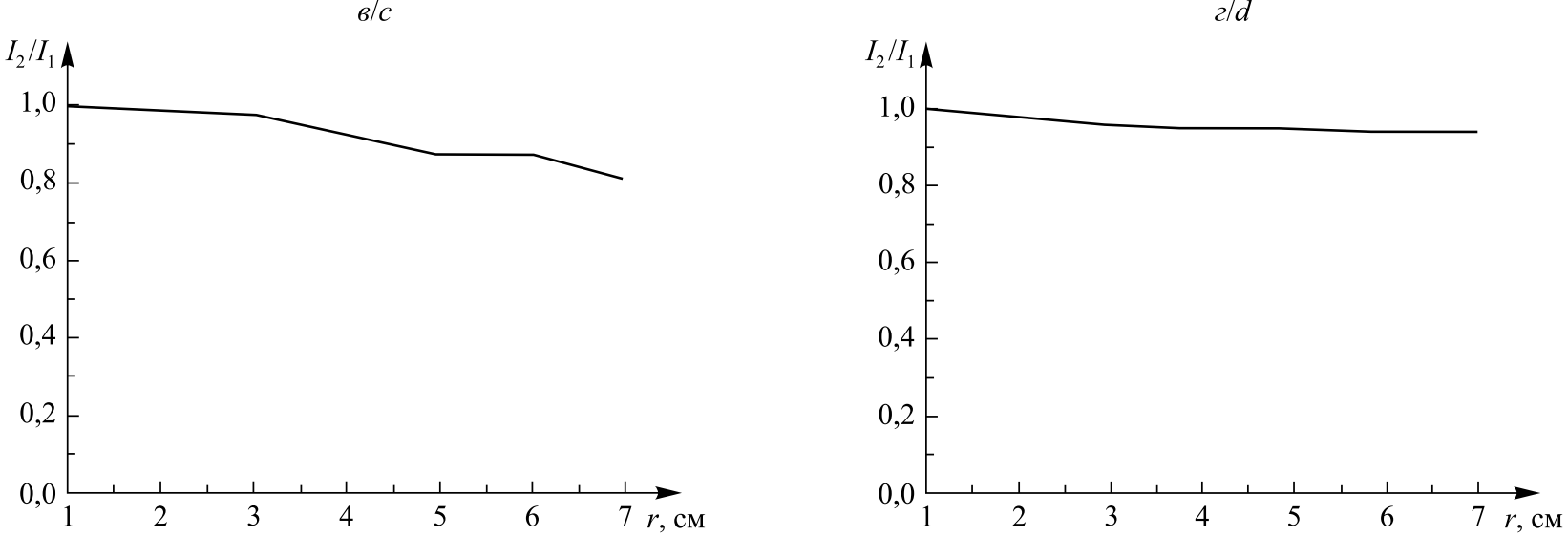

$P u c .4$. Отношение тока подвижного зонда к току неподвижного зонда при воздействии на алюминиевую мишень лазерного излучения плотностью мощности $3 \cdot 10^{8} \mathrm{~B}$ т $\mathrm{cm}^{2}$ при потенциале в промежутке мишень - сетка 20 В и различных потенциалах в промежутке сетка - подложка: $a-U_{2}=0 \mathrm{~B} ; \sigma-U_{2}=20 \mathrm{~B} ; в-U_{2}=50 \mathrm{~B} ; 2-U_{2}=100 \mathrm{~B}$

Fig. 4. The ratio of the current of a mobile probe to a current of a stationary probe at acting laser radiation on the aluminum target with a power density of $3 \cdot 10^{8} \mathrm{~W} / \mathrm{cm}^{2}$ at a potential in the target - grid interval of $20 \mathrm{~V}$ and at different potentials in the grid - substrate interval: $a-U_{2}=0 \mathrm{~V} ; b-U_{2}=20 \mathrm{~V} ; c-U_{2}=50 \mathrm{~V} ; d-U_{2}=100 \mathrm{~V}$

При появлении вторичной эмиссии регистрируется режим травления поверхности подложки [6], что способствует повышению адгезии. При дальнейшем увеличении кинетической энергии ионов за счет электрического поля в промежутке сетка - подложка (см. рис. 4, г) энергия ионов возрастает так, что ее хватает для имплантации ионов материала мишени в приповерхностную область подложки. При таких параметрах ионов потока сечение столкновения ионов потока с ионами подложки уменьшается. Как видно из рисунка, при этом наблюдается минимальная неравномерность плотности ионного потока на подложку $(\sim 5 \%)$.

Такой режим соответствует созданию псевдодиффузионного слоя на поверхности подложки материала лазерной мишени, что способствует увеличению адгезии.

\section{Заключение}

Проведенные эксперименты показали, что однородность плотности ионных потоков на подложку увеличенных размеров $\left(\sim 200 \mathrm{~cm}^{2}\right)$ в лазерно-плазменном источнике для нанесения нанопокрытий можно повысить, подавая на подложку ускоряющий потенциал (по отношению к сетке). Разница между плотностью ионного потока в центре подложки и на расстоянии 7 см от нее составляет $\sim 5 \%$. Это позволяет производить очистку поверхности подложки ионами материала лазерной мишени (вторичная эмиссия), создавать псевдодиффузионный слой материала мишени в приповерхностной области подложки, наносить на подложку материал лазерной мишени, причем все операции можно осуществлять последовательно, не разгерметизируя вакуумную камеру. Это обеспечит получение нанопокрытия с высокой адгезией и на подложках увеличенных размеров. 


\section{Библиографические ссылки}

1. Chrisey DB, Hubler GK, editors. Pulsed laser deposition of thin films. New York: John Wiley \& Sons; 1994. 648 p.

2. Bonelli M, Miotello A, Mosaner P. Pulsed lased deposition of diamondlike carbon films on polycarbonate. Journal of Applied Physics. 2003;93(2):859. DOI: 10.1063/1.1530725.

3. Vuoristo P, Tuominen J, Nurminen J. Laser coating and thermal spraying - process basics and coating properties. In: Proceedings of the International thermal spray conference and exhibition ITSC 2005; 2005 May 2-4; Basel, Switzerland. Düsseldorf: DVS-Verlag; 2005. p. 1270-1277.

4. Гончаров ВК, Козлова ЕИ, Пузырев МВ, Ступакевич ВЮ. Источник ионов алюминия с регулируемой энергией ионов. В: Попечиц ВИ, Дудчик ЮИ, Сенкевич ГА, редакторы. Прикладные проблемы оптики, информатики, радиофизики и физики конденсированного состояния. Материаль Четвертой Международной научно-практической конференции; 11-12 мая 2017 г.; Минск, Беларусь. Минск: [б. и.]; 2017. с. 209-211.

5. Гончаров ВК, Пузырев МВ, Ступакевич ВЮ. Физические процессы в лазерном источнике ионов алюминия с управляемой энергией для нанесения нанопленок. Журнал Белорусского государственного университета. Физика. 2017;3:79-87.

6. Goncharov VK, Puzyrev MV, Stupakevich VYu. Controlling charged-particle fluxes in the erosive laser plasma of a graphite target in vacuum. Journal of Engineering Physics and Thermophysics. 2018;91(4):1056-1062. DOI: 10.1007/s10891-018-1831-y.

\section{References}

1. Chrisey DB, Hubler GK, editors. Pulsed laser deposition of thin films. New York: John Wiley \& Sons; 1994. 648 p.

2. Bonelli M, Miotello A, Mosaner P. Pulsed lased deposition of diamondlike carbon films on polycarbonate. Journal of Applied Physics. 2003;93(2):859. DOI: 10.1063/1.1530725.

3. Vuoristo P, Tuominen J, Nurminen J. Laser coating and thermal spraying - process basics and coating properties. In: Proceedings of the International thermal spray conference and exhibition ITSC 2005; 2005 May 2-4; Basel, Switzerland. Düsseldorf: DVS-Verlag; 2005. p. 1270-1277.

4. Goncharov VK, Kozlova EI, Puzyrev MV, Stupakevich VYu. [A source of aluminum ions with regulated ion energy]. In: Popechits VI, Dudchik YuI, Senkevich GA, editors. Prikladnye problemy optiki, informatiki, radiofiziki i fiziki kondensirovannogo sostoyaniva. Materialy Chetvertoi Mezhdunarodnoi nauchno-prakticheskoi konferentsii; 11-12 maya 2017 g.; Minsk, Belarus' [Applied problems of optics, informatics, radiophysics and condensed matter physics. Proceedings of the $4^{\text {th }}$ International scientific and practical conference; 2017 May 11-12; Minsk, Belarus]. Minsk: [s. n.]; 2017. p. 209-211. Russian.

5. Goncharov VK, Puzyrev MV, Stupakevich VY. Physical processes in a laser source of aluminum ions with the controlled energy for nanofilm deposition. Journal of the Belarusian State University. Physics. 2017;3:79-87. Russian.

6. Goncharov VK, Puzyrev MV, Stupakevich VYu. Controlling charged-particle fluxes in the erosive laser plasma of a graphite target in vacuum. Journal of Engineering Physics and Thermophysics. 2018;91(4):1056-1062. DOI: 10.1007/s10891-018-1831-y. 